\title{
Hypothesen über die Bildung von Kaolinit in Böden der Schweiz
}

\begin{abstract}
Zusammenfassung
Üblicherweise wird angenommen, daß die Bildung von Kaolinit in Bödez ausschließlich an das Vorherrschen warmfeuchter Klimabedingungen sowie einer intensiven Perkolation des Bodens gebunden sein muß. Die von uns durchgeführten Untersuchungen an ausgewählten Bodenprofilen im schweizerischen Mittelland, im Jura und in den Alpen belegen, daß Kaolinit in einigen Fällen nachgewiesen werden kann und dies auch unter temperierten bis subalpinen Klimabedingungen sowie in vergleichsweise jungen Böden. Entscheidend für eine rezente Kaolinitbildung scheint jeweils der Wasserhaushalt des Bodens zu sein: unsere Ergebnisse erlauben die Hypothese, daß eine gehemmte Perkolation oder der Einfluß des Grundwassers die Kristallisation von Kaolinit begünstigen.
\end{abstract}

\section{Summary}

It is usually assumed that the formation of kaolinite in soils is exclusively bound to warm and humid climatic conditions and to an intensive percolation rate. The investigations performed on selected soil profiles in the Swiss plateau, the Jura and the Alps show that kaolinite is detectable under special circumstances. Kaolinite can obviously precipitate also in soils of temperate to subalpine climate zones. Thereby, the hydrologic situation of the soil seems to be crucial for possible kaolinite formation: our results allow the hypothesis that groundwater or a restrained percolation rate favour the crystallization of kaolinite.

\section{Einleitung}

Kaolinit ist im wesentlichen ein 1:1-Schichtsilikat. 1:1-Minerale haben eine regelmäßige Abfolge von je einer Oktaeder- und einer Tetraederschicht. Die Tetraeder- und Oktaederschichten sind über gemeinsame O-Ionen verbunden. Kaolinit setzt sich dabei aus einer Silikatschicht $\left(\mathrm{Si}_{4} \mathrm{O}_{10}\right)^{4-}$ und einer Gibbsitschicht mit $(\mathrm{OH})_{6} \mathrm{Al}_{4}(\mathrm{OH})_{2} \mathrm{O}_{4}$ zusammen sowie aus Randzonen, bestehend aus $\mathrm{Al}(\mathrm{OH})_{3}$ und $\mathrm{SiO}_{2}$ (STUMm 1992). Die Entstehung von Kaolinit kann prinzipiell über zwei verschiedene Wege erfolgen, nämlich über die Verwitterung von Primärmineralien (wie Feldspäte oder Schichtsilikate) während der Pedogenese oder über hydrothermale Prozesse. Die Hydrolyse von Feldspäten ist ein zentraler Aspekt der Kaolinitbildung. Entsprechende, inkongruente Lösungsreaktionen von K-Feldspäten können beispielsweise wie folgt umschrieben werden (BIRKELAND 1984):

$$
\begin{aligned}
& 4 \mathrm{~K}_{\mathrm{x}} \mathrm{Na}_{1-\mathrm{x}} \mathrm{AlSi}_{3} \mathrm{O}_{8}+4 \mathrm{H}^{+}+18 \mathrm{H}_{2} \mathrm{O}= \\
& \mathrm{Al}_{4}\left[\mathrm{Si}_{4} \mathrm{O}_{10}\right][\mathrm{OH}]_{8}+8 \mathrm{H}_{4} \mathrm{SiO}_{4}+4 \mathrm{xK}^{+}+4(1-\mathrm{x}) \mathrm{Na}^{+}
\end{aligned}
$$

Zum Teil bildet sich Kaolinit auch aus pyroklastischen Gesteinen sowie aus Biotit und Muskovit (ESTEOULECHOUX \& BLANCHET 1987). Aus theoretischen und experimentellen Arbeiten zur Tonmineral- und Kaolinitgenese führt HEIM (1990) die folgenden Faktoren auf, die die Bildung von Kaolinit begünstigen oder sogar als notwendige Voraussetzungen betrachtet werden müssen:

- tiefe $\mathrm{Si}(\mathrm{OH})_{4}$-Konzentrationen in der Verwitterungslösung, da die Polymerisation sonst zu rasch erfolgt

- sehr langsame Al-Hydroxidfällung, da sich sonst Bayerit statt Gibbsit $\left(=\gamma-\mathrm{Al}[\mathrm{OH}]_{3}\right)$ bildet, und nur dieser besitzt O-Schichten, die sich als Matrize für die Genese von T-Schichten eignen

- niedriges Si/Al-Verhältnis in der Verwitterungslösung, da sich sonst Dreischichtminerale bilden können

- ein saures Milieu mit pH-Werten etwa um 5

- möglichst geringe Anteile von potentiellen Zwischenschicht-Kationen, wobei besonders $\mathrm{K}^{+}$und $\mathrm{Ca}^{2+}$ die Genese von Zweischichtmineralen verhindern können.

HEIM (1990) und MURRAY \& KELLER (1993) postulieren, $\mathrm{da} ß$ das Vorkommen von Kaolinit auf dem Festland in Zusammenhang mit der intensiven Verwitterung unter tropischen und subtropischen Klimabedingungen steht. Gemäß diesen Autoren haben hohe Niederschläge und ein tiefliegender Grundwasserspiegel zur Folge, daß das Bodensickerwasser und die darin gelösten Komponenten schnell durch den Boden perkolieren. In subtropischen Gebieten konnte BARSHAD (1966) aufgrund mehrerer hundert Oberflächen-Bodenanalysen eine klare Korrelation zwischen Niederschlagsmenge, Ausgangs-

Markus Egli, Dr. ${ }^{1}$, Peter Fitze, Prof. Dr. ${ }^{2}$, Sonja Gehrig, dipl. Geographin², Matthias Achermann, dipl. Geograph

${ }^{1}$ Kant. Amt für Umweltschutz, Klosterstraße 31, 6002 Luzern

2 Geographisches Institut, Universität Zürich Irchel, Winterthurerstraße 190, 8057 Zürich 
felsische Ausgangsgesteine

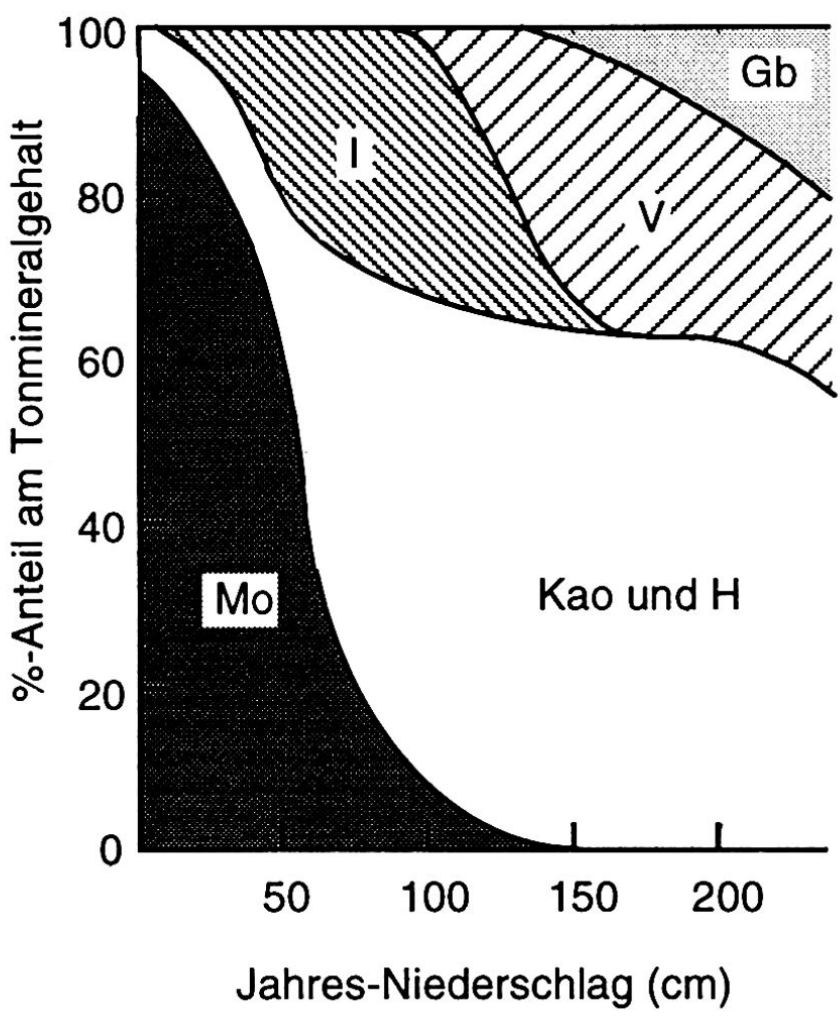

mafische Ausgangsgesteine

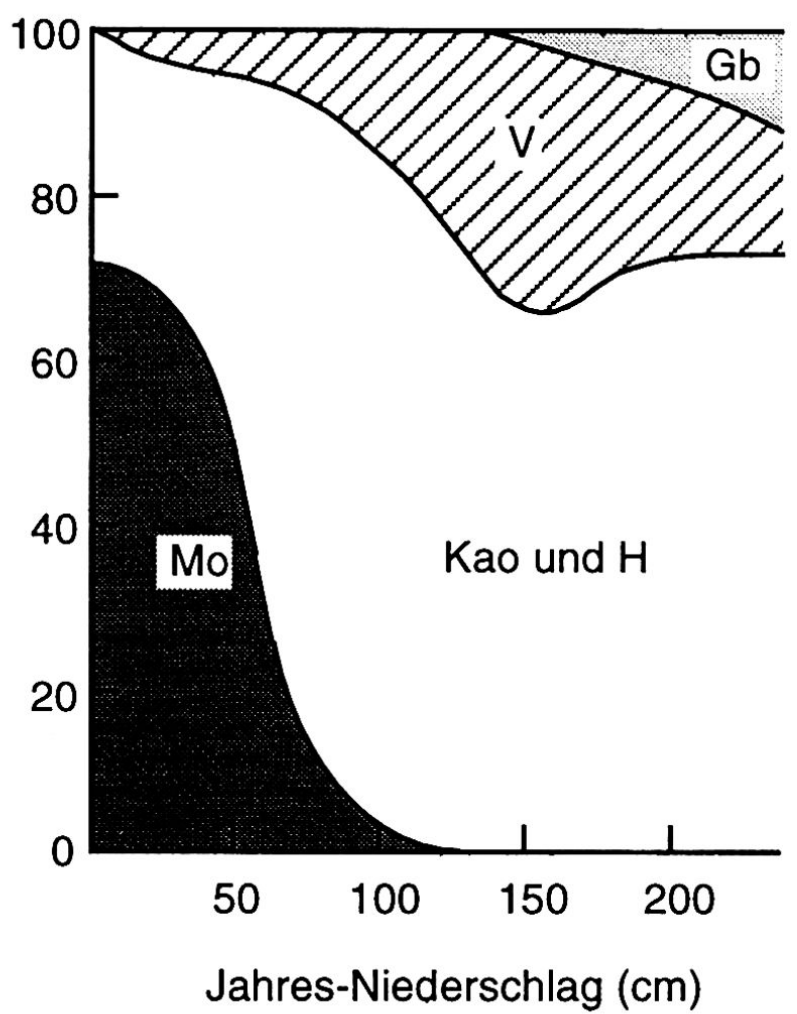

Fig. 1. Relativer Mineralgehalt (von oberflächennahen Bodenproben) als Funktion des Niederschlags (Mo $=$ Montmorillonit, $\mathrm{KaO}=$ Kaolinit, $\mathrm{H}=$ Halloysit, $\mathrm{I}=\mathrm{Illit}, \mathrm{V}=$ Vermiculit, $\mathrm{Gb}=$ Gibbsit) und in Abhängigkeit des Ausgangsgesteins. Die Darstellung bezieht sich auf subtropische Bedingungen.

gestein (mafische oder felsische Ausgangsgesteine) und der Mineralogie erkennen. Daraus geht lehrbuchmäßig hervor, daß höhere Niederschläge und eine größere Auswaschung die Bildung von Kaolinit sehr stark intensivieren (Fig. 1).

Die hydrothermale Interaktion von Wasser mit kristallinem Gestein in Tiefen mit einer Temperatur bis etwa $250^{\circ} \mathrm{C}$ ist eine weitere Möglichkeit der Kaolinitgenese. Der dabei entstehende Kaolinit ist meist von großer Reinheit, ganz im Gegensatz zum pedogenetisch entstandenen Kaolinit, wo Nebengemengteile wie Fe-Oxide und -Hydroxide als Verunreinigungsprodukte auftreten. Der Prozeß der hydrothermalen Bildung ist im Prinzip nichts anderes als eine Extension der Verwitterungsprozesse. Die Reaktionsprodukte sind durch dieselben Parameter - nämlich die Umgebungschemie, Zeit und Temperatur - gesteuert (VELDE 1992).

Allgemein bekannt ist auch die Tatsache, daß Kaolinit nicht nur in tropischen bis subtropischen Klimaten vorzufinden ist, sondern auch in Böden gemäßigter bis subpolarer Klimaregionen (in meist eher geringen Mengen) vorhanden sein kann. Die Prozesse, welche die Kaolinitbildung unter solchen Rahmenbedingungen steuern, sind hingegen wenig untersucht. Die von uns durchgeführten Untersuchungen umspannen somit die Frage, unter welchen pedologischen Bedingungen Kaolinit auch in Böden der Schweiz vorzufinden ist.

\section{Untersuchungsgebiete}

Mit der entsprechenden Auswahl der Untersuchungsgebiete wurde versucht, einen groben - jedoch sicherlich nicht vollständigen - Querschnitt der naturräumlichen Einheiten der Schweiz zu erfassen. Die Untersuchungsgebiete befinden sich im schweizerischen Mittelland, im Rheintal (nah bei Basel), im Jura und in den Alpen. Profildaten aus folgenden Lokalitäten standen dabei zur Verfügung:

- Rheintal: Möhlin (Standorte Seeliboden und Chräbis mit den Koordinaten 633 750/269575 bzw. $631325 /$ 270275)

- Jura: bei Oberbuchsiten, mit den Koordinaten $623300 / 239360$

- Luzerner Mittelland: Lokalität Buchen, nah bei Wolhusen, mit den Koordinaten 648 275/213950

- Alpen: Oberengadin (Isola-Delta, mit den Koordinaten $776703 / 143163$ )

\section{Möhlin}

Die morphologische Struktur des Untersuchungsgebietes wird geprägt durch Lockergesteine, deren Ablagerung während der pleistozänen Vereisungsphase statt- 
fand. Neben Flußschottern, die als Terrassen ausgebildet sind, treten geringmächtige moränale Sedimente und Formen auf, deren Bildung auf den weitesten Vorstoß eines alpinen Gletschers bis nach Möhlin zurückgeht. Während des Würm-Glazials wurde feinkörniges Material aus den höherliegenden Terrassen ausgeweht und in Form von Löss in der näheren Umgebung von Möhlin wieder abgelagert (KÜHNEN 1984). Parabraunerden, die sich nach der Maximalausdehnung der Gletscher bilden konnten (ca. $20000 \mathrm{yBP}$ ), prägen zum größten Teil die pedologische Situation.

Die jährliche Niederschlagsmenge beträgt heute ca. $1000 \mathrm{~mm}$ pro Jahr, und die Jahresmitteltemperatur beläuft sich auf rund $9,3^{\circ} \mathrm{C}$.

\section{Jura (Oberbuchsiten)}

Bei den untersuchten Böden handelt es sich nicht um rezente, sondern um relikte Böden. Die untersuchten Böden lassen sich als Terra fusca umschreiben. Diese Böden bildeten sich auf Kalkgestein, vorwiegend im Eozän (37-58 Mio. yBP) unter subtropischen bis tropischen und humiden Bedingungen. Die dabei entstandene Terra fusca (Kalksteinbraunlehm) stellt ein Endstadium der Bodenbildung auf hochprozentigen Kalksteinen dar.

Unter den heutigen klimatischen Verhältnissen fallen pro Jahr ca. 1100 mm Niederschlag. Die jährliche Durchschnittstemperatur beläuft sich auf ca. $8,5^{\circ} \mathrm{C}$.

\section{Luzerner Mittelland}

Der Untersuchungsstandort befindet sich in der Nähe von Wolhusen (Lokalität Buchen) auf landwirtschaftlich bearbeitetem Gelände. Das Ausgangsmaterial für die Bodenbildung besteht einerseits aus geringmächtigen Moränensedimenten, welche beim größten Vorstoß der Gletscher während der letzten Eiszeit abgelagert wurden, und andererseits aus Sandstein- und Mergellagen der Oberen Süßwassermolasse sowie aus Konglomeraten (Molassenagelfluh) des Napffächers. Die Böden können als (pseudovergleyte) Braunerden bezeichnet werden.

Die mittlere Jahrestemperatur beträgt ca. $8^{\circ} \mathrm{C}$ und die durchschnittliche jährliche Niederschlagsmenge ca. $1100 \mathrm{~mm}$.

\section{Isola-Delta}

Das Isola-Delta befindet sich am Südostufer des Silsersees. Das an das Delta angrenzende Val Fedoz besteht geologisch aus granodioritischen Hellglimmeraugengneisen, flaserigen Grünschiefern, Metagabbros, Metadioriten und aus metapelitischen Bändergneisen mit Metakarbonaten und Amphibolen sowie aus Triassedimenten (SPILlMANN 1993). Der jährliche mittlere Niederschlag beläuft sich für das Isola-Delta auf ca. 1000 $\mathrm{mm}$, und die durchschnittliche Jahrestemperatur beträgt ca. $1,6^{\circ} \mathrm{C}$.

\section{Methoden}

Die Entnahme der Bodenproben erfolgte im Falle von Möhlin und dem Luzerner Mittelland mit einem HUMAX-Bohrgerät. Im Isola-Delta wurden Probebohrungen mit einem Rammsondierer durchgeführt, während in Oberbuchsiten (Jura) das Bodenmaterial direkt aus einem vorliegenden Aufschluss entnommen werden konnte.

Die bodenchemischen und tonmineralogischen Bestimmungen im Labor wurden am Probenmaterial mit einem Durchmesser $<2 \mathrm{~mm}$ durchgeführt. Die Bestimmung des pedogenen Fe-Anteils im Boden erfolgte über einen $\mathrm{HNO}_{3}$ - (Standorte Möhlin und Luzerner Mittelland) oder Dithionitauszug (Standorte Jura und IsolaDelta). Dithionitlösliches Fe wurde in einer Na-Dithionit-Citrat-Bicarbonat-Lösung analysiert. Für Mittellandböden haben gemäß FITZE et al. (1989) der $\mathrm{HNO}_{3}$ und Dithionitauszug in etwa die gleiche Extraktionsstärke bezüglich des Elementes Fe. Der Boden-pH wurde in einer Bodensuspension, bestehend aus 1 Teil getrockneter Feinerde und 2,5 Teilen 0,01 $\mathrm{M} \mathrm{CaCl}_{2-}$ Lösung, gemessen. Der Fe-Gehalt wurde mittels Atomabsorptionsspektroskopie analysiert. Die Korngrößenfraktionierung von Feinmaterial wurde nach Humuszerstörung und einer Ultraschallbehandlung durch Siebung und Sedimentation (Aerometer) analysiert. Für die Proben des Isola-Deltas mußte aufgrund von Materialknappheit die Korngrößenverteilung geschätzt werden. Die Identifizierung der Tonminerale der Feinerde $(<2 \mathrm{~mm}$, feinst gemahlen) und der Tonfraktion $(<2 \mu \mathrm{m})$ sowie deren halbquantitative Bestimmung erfolgten mittels Röntgendiffraktometeranalyse am Geotechnischen Institut der ETHZ sowie am Institut für Mineralogie und Petrographie der ETHZ. Zur Analyse gelangten karbonatfreie Proben. Für die Bestimmung der mineralogischen Probenzusammensetzung der Tonfraktion mußte vorgängig die Feinerde mit Ultraschall dispergiert und anschließend die Fraktion $<2 \mu \mathrm{m}$ abgetrennt werden. Die Altersbestimmung der Böden des Isola-Deltas erfolgte einerseits am organischen Material mittels der Radiokarbonmethode $\left({ }^{14} \mathrm{C}\right.$-Alter $)$. Andererseits wurden bei fehlenden ${ }^{14} \mathrm{C}$-Daten die Alter der betreffenden Schichten mit Hilfe der Pollenfrequenz berechnet. Die für die ${ }^{14} \mathrm{C}$-Altersbestimmung erforderliche Präparierung und Aufbereitung des Probenmaterials erfolgte im Radiokarbonlabor des Geographischen Institutes der Universität Zürich (GIUZ). Die anschließende Datierung wurde mittels der AMS-Technik (accelerator mass spectrometry) auf dem Tandembeschleuniger des ITP (Institut für Teilchenphysik) der ETH Hönggerberg durchgeführt. Intensive Sickerwasseranalysen wurden in den Profilen Seeliboden und Chräbis (beide Standort Möhlin) durchgeführt. Die dabei benötigte Methodik ist ausführlich in EGLI (1995) diskutiert. Zur Simulation der Sickerwasser- bzw. Grundwasserzusammensetzung wurde für den Probestandort Isola am Bodenmaterial ein Wasserextraktionsverfahren angewendet $(48 \mathrm{~h}$ vorbefeuchten, 2 h Extraktion, vgl. WINISTÖRFER 1993). 


\section{Ergebnisse und Diskussion}

\section{Möhlin}

Die Lokalität Seeliboden befindet sich auf reinem Lößsubstrat und ist bis in eine Tiefe von ca. 3 m entkarbonatisiert. Der Boden der Lokalität Chräbis besteht aus zwei verschiedenen Substraten. Der C-Horizont (kalkhaltiger Schotter) befindet sich dabei in einer Tiefe von rund $110 \mathrm{~cm}$. Bedingt durch die Erosionsaktivitäten des Rheins seit der letzten Eiszeit, wurde vorverwitterter Löß (das gleiche Ausgangsmaterial wie bei Seeliboden) auf dem Schotter abgelagert und teilweise mit ihm vermischt. Aus diesem Material entwickelte sich danach der eigentliche Boden. An beiden Standorten handelt es sich um saure Parabraunerden, was unter anderem am Profilverlauf des pedogenen Fe erkennbar ist (Tab. 1 und 2). Der damit einhergehende Proze $\beta$ der Tonverlagerung scheint aber reliktischer Natur zu sein. Durch die Tonverlagerung entstand im Boden die typische Ausbildung eines Eluvialhorizontes (mit erniedrigtem Tonfraktionsgehalt, vgl. Tab. 1 und 2), gefolgt in etwas größerer Tiefe von einem Illuvialhorizont. Beide untersuchten Böden sind im oberen Profilbereich stark sauer mit $\mathrm{pH}$-Werten zwischen 3,8 und 4,2. Eine aktive Tonverlagerung findet im allgemeinen in einem $\mathrm{pH}$-Bereich von 5 bis ca. 6,5 statt. Die Böden können als normal perkoliert taxiert werden, da Pseudovergleyungen, wenn überhaupt, nur sehr schwach erkennbar waren. Tonmineralogische Untersuchungen ergaben, daß beide Stand- orte über den jeweiligen Profilverlauf eine sehr ähnliche mineralogische Zusammensetzung aufweisen. Das mineralogische Spektrum wird beherrscht durch Quarz, Plagioklas, Alkali-Feldspäte, Chlorit, Montmorillonit, Smectit und Illit (nur Seeliboden). Kaolinit und Jurbanit werden nur in Spurenanteilen (max. 5\%-Vol., nur Standort Chräbis) vermutet. Die Bedingungen gemäß HEIM (1990) für eine Kaolinitbildung wären bei beiden Standorten theoretisch erfüllt. Eine Ausnahme bildet der $\mathrm{pH}$-Wert der Sickerwasserlösung in den oberen Bodenhorizonten, wo im allgemeinen Werte zwischen 3,8 und 4 gemessen wurden (EGLI 1995). In tieferliegenden Bodenhorizonten steigt der pH-Wert jedoch gegen 5: Löslichkeitsberechnungen zeigen, da $\beta$ auch hier eine rezente Kaolinitbildung nicht manifestierbar ist. Ob eine Lösung im Gleichgewicht mit einer Festphase steht, ergibt sich über den Sättigungsindex $(\Omega)$, welcher durch die Gleichung

$$
\Omega=\log Q \mathrm{p}-\log \mathrm{K},
$$

mit $\mathrm{Qp}$ als Ionen-Aktivitätsprodukt und $\mathrm{K}$ als Löslichkeits-Gleichgewichtskonstante definiert ist. Eine Übersättigung der Bodenlösung bezüglich der Festphase herrscht, wenn $\Omega>0$, bzw. Untersättigung, wenn $\Omega<0$ ist. Die Bodenlösung war gegenüber der Festphase Kaolinit in allen Bodenhorizonten stark untersättigt $(-8,4<\Omega<-1,6$, basierend auf thermodynamischen Daten von PLUMMER et al. 1976 und SIGG \& STUMM 1994). Diese Untersättigung war in den oberen Bodenhorizonten besonders ausgeprägt (vgl. EGLI 1995).

Tabelle 1 Bodeneigenschaften der Lokalität Chräbis

\begin{tabular}{|c|c|c|c|c|c|c|c|}
\hline $\begin{array}{l}\text { Tiefe } \\
(\mathrm{cm})\end{array}$ & $\begin{array}{l}\text { Horizont- } \\
\text { bezeichnung }\end{array}$ & $\begin{array}{l}\mathrm{pH} \\
\left(\mathrm{CaCl}_{2}\right)\end{array}$ & $\begin{array}{l}\mathrm{Fe}_{\mathrm{HNO}_{3}{ }^{\mathrm{d}}} \\
\mathrm{g} \mathrm{kg}^{-1}\end{array}$ & $T(\%)$ & $\begin{array}{c}\text { Körnung a } \\
U(\%)\end{array}$ & $S(\%)$ & Mineralogie $^{b}$ \\
\hline 5 & $\mathrm{Ah} / \mathrm{E}$ & 3,7 & 7,1 & 6,5 & 21,7 & 71,8 & Qz, Kfsp, Plag, Chl, Mo, S, ${ }^{c}\{K a o\}$ \\
\hline 45 & It $(B w)$ & 4,2 & 13,2 & 18,0 & 21,0 & 61,0 & Qz, Kfsp, Plag, Chl, Mo, S, \{Ju\} \\
\hline 65 & It $(B w)$ & 4,1 & 14,8 & 15,0 & 19,0 & 66,0 & Qz, Kfsp, Plag, Chl, Mo, S, \{Ju\} \\
\hline 113 & C & 6,9 & 6,4 & 5,0 & 9,0 & 86,0 & \\
\hline 170 & C & 7,5 & 4,4 & 1,4 & 2,6 & 96,0 & \\
\hline
\end{tabular}

a $T=$ Tonfraktion $(<2 \mu \mathrm{m}), U=$ Siltfraktion $(2-63 \mu \mathrm{m})$ und $S=$ Sandfraktion $(63-2000 \mu \mathrm{m})$

b $\mathrm{Qz}=$ Quarz, Kfsp = Kali-Feldspat, Plag = Plagioklas, Chl = Chlorit, Mo = Montmorillonit, $\mathrm{S}=\mathrm{Smectit}$, Kao = Kaolinit, Ju = Jurbanit

${ }^{c}\{\}$ in Spuren vorhanden bzw. vermutet

a $\mathrm{Fe}_{\mathrm{HNO}}$ : $\mathrm{HNO}_{3}$-Auszug

Tabelle 2 Bodeneigenschaften der Lokalität Seeliboden

\begin{tabular}{|c|c|c|c|c|c|c|c|}
\hline $\begin{array}{l}\text { Tiefe } \\
\text { (cm) } \\
\end{array}$ & $\begin{array}{l}\text { Horizont- } \\
\text { bezeichnung }\end{array}$ & $\begin{array}{l}\mathrm{pH} \\
\left(\mathrm{CaCl}_{2}\right) \\
\end{array}$ & $\begin{array}{l}\mathrm{Fe}_{\mathrm{HNO}_{3}}{ }^{\mathrm{c}} \\
\mathrm{g} \mathrm{kg}^{-1}\end{array}$ & $\mathrm{~T}(\%)$ & $\begin{array}{c}\text { Körnunga } \\
U(\%)\end{array}$ & $\mathrm{S}(\%)$ & Mineralogie $^{b}$ \\
\hline 5 & Ah & 3,8 & 9,5 & 3,0 & 21,0 & 76,0 & Qz, Kfsp, Plag, Chl, Mo, Mu, S, I \\
\hline 42 & (It) $\mathrm{Bw}$ & 3,8 & 11,1 & 16,0 & 60,0 & 24,0 & Qz, Kfsp, Plag, Chl, Mo, Mu, S, I \\
\hline 85 & (It) $\mathrm{Bw}$ & 4,1 & 13,1 & 16,0 & 32,9 & 51,1 & Qz, Kfsp, Plag, Chl, Mo, Mu, S, I \\
\hline 122 & (It) Bw & 4,1 & 12,5 & 17,0 & 63,0 & 20,0 & Qz, Kfsp, Plag, Chl, Mo, Mu, S, I \\
\hline 172 & Bw & 4,0 & 10,7 & 13,0 & 55,0 & 32,0 & Qz, Kfsp, Plag, Chl, Mo, Mu, S, I \\
\hline 227 & Bw & 4,4 & 13,7 & 17,0 & 68,0 & 15,0 & \\
\hline 345 & C & 7,5 & 9,8 & 5,0 & 81,0 & 14,0 & \\
\hline
\end{tabular}

a $T=$ Tonfraktion $(<2 \mu \mathrm{m}), \mathrm{U}=$ Siltfraktion $(2-63 \mu \mathrm{m})$ und $S=$ Sandfraktion $(63-2000 \mu \mathrm{m})$

${ }^{\mathrm{b}} \mathrm{Qz}=$ Quarz, Kfsp = Kali-Feldspat, Plag = Plagioklas, Chl = Chlorit, Mo = Montmorillonit, Mu = Muskovit, $\mathrm{S}=\mathrm{Smectit}$, I = Illit

c $\mathrm{Fe}_{\mathrm{HNO}}$ : $\mathrm{HNO}_{3}$-Auszug 


\section{Jura (Oberbuchsiten)}

Die Terra-fusca-Vorkommen befinden sich im Bereich der Siderolithformationen, die durch Erosionsprozesse nach deren Bildung und späterer Einsedimentation wiederum freigelegt wurden. Eine Terra fusca in ihrem ursprünglichen Aufbau mit der Horizontabfolge Ah $\mathrm{Bw}-\mathrm{Cc}$ ist nirgendwo mehr vorzufinden. Ursprüngliches Bw-Material findet sich bevorzugt in Karsttaschen. Die tertiären Verwitterungsprodukte des Bw-Horizontes zeichnen sich durch intensive Umlagerung, unregelmäßiges Vorkommen, unterschiedliche Mächtigkeiten und Zusammensetzung aus. Das sehr tonreiche Residualmaterial von In-situ-Profilen weist eine braungelbe bis rote Farbe auf und ist meist vollkommen entkarbonatisiert. Die Profildaten (vgl. pH-Werte, Tab. 3) zeigen, daß das ursprüngliche Bw-Material sekundär aufgekalkt wurde, sei es durch direkt eingelagertes Karbonatmaterial oder durch Sicker- und Hangwasser. In den Verwitterungsprodukten finden sich Ansammlungen von Bohnerz, das die Basis der Eisenverhüttung im Jura bildete. Der Bolus, d.h. die Verwitterungsprodukte (ohne Bohnerz), wurde bezüglich seiner Mineralogie untersucht. Der karbonatfreie Bolus setzt sich im wesentlichen aus Kaolinit (ca. 40-50\%), Quarz (ca. 20-30\%) und Goethit (ca. 10-30\%) zusammen.

Diese Terra-fusca-Vorkommen sind dem Kimmeridge des Malmkalkes aufgelagert. Es stellt sich nun die Frage, ob der hohe Kaolinitgehalt dieser Böden nur eine Folge der Karbonatauswaschung ist und somit nichts anderes als eine passive Anreicherung der Residuen darstellt. Böden auf vergleichbarem Ausgangsgestein (Kimmeridge, Malmkalk) im Vallée de Joux / Mont Tendre zeigen auf, daß der Kaolinitgehalt in der karbonatfreien Fraktion des Ausgangsgesteins und des Bodens im Bereich von $<5$ bis ca. $15 \%$ schwankt (BGS 1979; POCHON 1979). Der gleichförmige Verlauf des Kaolinitgehaltes in der karbonatfreien Fraktion dieser Böden (Vallée de Joux / Mont Tendre) deutet darauf hin, daß mindestens seit der letzten Eiszeit keine signifikante, aktive Kaolinitbildung erfolgte. Aus diesen Überlegungen kann gefolgert werden, daß die hohen Kaolinitgehalte der Terra fusca nicht nur eine Rückstandsanreicherung von karbonatfreiem Material (Residuen) darstellen, sondern dass die Kaolinitbildung zu einem beträchtlichen Teil auf aktive Mineralumwandlungsprozesse zu jener Zeit (Eozän) zurückgeführt werden kann. Überschüttete präwürmeiszeitliche Bodenprofile (Vallé de Joux; BGS 1979, POCHON 1979), deren Alter nicht näher datiert worden sind, scheinen ebenfalls eine gewisse Phase der Kaolinitbildung durchlaufen zu haben: der Illitgehalt geht vom R-Horizont (Felsunterlage) zum IIB-Horizont relativ stark zurück, während der Kaolinitgehalt sich reziprok dazu verhält. Gemäß SCHWER (1994) kann die Umwandlung von Illit über die Bildung des Zwischenprodukts Smectit (durch den Einbau von $\mathrm{Ca}^{2+}, \mathrm{Na}^{+}$, $\mathrm{Si}^{4+}, \mathrm{Mg}^{2+}, \mathrm{Fe}^{2+}$ und $\mathrm{H}_{2} \mathrm{O}$ ) und durch eine verstärkte Desilifizierung und Versauerung zum Endprodukt Kaolinit und Quarz erfolgen. POCHON (1979) vermutete, daß diese fossilen, präwürmeiszeitlichen Böden aufgrund ihres hohen Kaolinitgehaltes ebenfalls als Terra fusca gedeutet werden können.

\section{Luzerner Mittelland}

Der Boden der Lokalität Buchen besteht aus mehreren Ausgangssubstraten. Der eigentliche C-Horizont (Molassenagelfluh) befindet sich in ca. $180 \mathrm{~cm}$ Tiefe. Darüber befinden sich verwitterte Sedimente der Oberen Süsswassermolasse, welche zum Teil mit der obersten Schicht, den Moränenablagerungen, vermischt sind. Der vorgefundene Boden kann als tiefgründige, pseudovergleyte Braunerde angesprochen werden. Durch die landwirtschaftliche Bearbeitung wurden die obersten $30 \mathrm{~cm}$ immer wieder durchmischt und homogenisiert. Der atypische Verlauf des pH-Wertes (Tab. 4), insbesondere in den oberen Bodenhorizonten, weist darauf hin, da $\beta$ der Boden zeitweise aufgekalkt wurde. Die gemessenen Kalkgehalte in den obersten Bodenschichten (nicht in Tab. 4 aufgeführt) bestätigen diese Vermutung. Der Boden ist ab einer Tiefe von ca. $100 \mathrm{~cm}$ von gestautem Sickerwasser geprägt, was zur Ausbildung von Horizonten mit starker Rostfleckung infolge periodischer Vernässung und Durchlüftung geführt hat.

Tabelle 3 Bodeneigenschaften der Lokalität Oberbuchsiten

\begin{tabular}{rlllllll}
\hline $\begin{array}{l}\text { Tiefe } \\
(\mathrm{cm})\end{array}$ & $\begin{array}{l}\text { Horizont- } \\
\text { bezeichnung }\end{array}$ & $\begin{array}{l}\mathrm{pH} \\
\left(\mathrm{CaCl}_{2}\right)\end{array}$ & $\begin{array}{l}\mathrm{Fe}_{\mathrm{d}}{ }^{\mathrm{c}} \\
\mathrm{g} \mathrm{kg}^{-1}\end{array}$ & $\mathrm{~T}(\%)$ & $\begin{array}{c}\text { Körnung } \\
\mathrm{U}(\%)\end{array}$ & $\mathrm{S}(\%)$ & Mineralogie $^{\mathrm{b}}$ \\
\hline 45 & $\mathrm{bBw}, \mathrm{k}$ & 7,4 & 25,5 & & & & Kao, Qz, G, Cc \\
55 & bBw, k & 7,5 & 25,2 & 23,7 & 31,8 & 44,5 & Kao, Qz, G, Cc \\
65 & bBw, k & 7,5 & 23,7 & 15,3 & 19,5 & 65,2 & Kao, Qz, G, Cc \\
150 & bBw, k & 7,6 & 30,4 & 26,7 & 25,4 & 47,9 & Kao, Qz, G, Cc \\
\hline
\end{tabular}

a $T=$ Tonfraktion $(<2 \mu \mathrm{m}), \mathrm{U}=$ Siltfraktion $(2-63 \mu \mathrm{m})$ und $S=$ Sandfraktion $(63-2000 \mu \mathrm{m})$

b $\mathrm{Qz}=$ Quarz, $\mathrm{Kao}=$ Kaolinit, $\mathrm{G}=$ Goethit, $\mathrm{Cc}=$ Calcit

c $\mathrm{Fe}_{\mathrm{d}}$ : Dithionit-Auszug (Analysewerte beziehen sich auf karbonathaltige Probe) 
Tabelle 4 Bodeneigenschaften der Lokalität Buchen

\begin{tabular}{|c|c|c|c|c|c|c|c|}
\hline $\begin{array}{l}\text { Tiefe } \\
(\mathrm{cm})\end{array}$ & $\begin{array}{l}\text { Horizont- } \\
\text { bezeichnung }\end{array}$ & $\begin{array}{l}\mathrm{pH} \\
\left(\mathrm{CaCl}_{2}\right)\end{array}$ & $\begin{array}{l}\mathrm{Fe}_{\mathrm{HNO} 3^{\mathrm{d}}} \\
\mathrm{g} \mathrm{kg}^{-1}\end{array}$ & $T(\%)$ & $\begin{array}{c}\text { Körnunga } \\
\cup(\%)\end{array}$ & $\mathrm{S}(\%)$ & Mineralogie $^{b}$ \\
\hline 5 & Ah, $p$ & 4,8 & 9,6 & & & & \\
\hline 15 & Ah, $p$ & 5,0 & n. b. & 8,0 & 31,5 & 60,5 & Qz, Kfsp, Plag, Chl, Mo, Mu, c $\{l\}$ \\
\hline 35 & Bw & 5,0 & 8,6 & 12,0 & 26,0 & 62,0 & \\
\hline 57 & Bw & 5,0 & 7,7 & 12,5 & 27,5 & 59,0 & \\
\hline 83 & Bw & 5,0 & 6,9 & 10,0 & 33,5 & 56,5 & Qz, Kfsp, Plag, Chl, Mo, Mu \\
\hline 94 & $B w, g$ & 5,0 & 6,8 & & & & \\
\hline 110 & $\| \mathrm{Bw}, \mathrm{g}$ & 4,8 & 6,3 & 20,0 & 42,0 & 38,0 & Qz, Plag, Kfsp, Chl, Mu \\
\hline 172 & II BCgg & 5,$4 ; 6,1^{\star \star}$ & 9,4 & 14,5 & 28,5 & 57,0 & $\mathrm{KaO}^{\star}, \mathrm{Qz}^{\star}$, Lep*, Qz, Plag, Kfsp, Chl, Mu \\
\hline 180 & III C & $>7$ & n. b. & & & & \\
\hline
\end{tabular}

a $T=$ Tonfraktion $(<2 \mu \mathrm{m}), U=$ Siltfraktion $(2-63 \mu \mathrm{m})$ und $S=$ Sandfraktion $(63-2000 \mu \mathrm{m})$

${ }^{\mathrm{b}} \mathrm{Qz}=$ Quarz, Kao = Kaolinit, Lep $=$ Lepidokrokit, Kfsp = Kali-Feldspat, Plag = Plagioklas, Chl = Chlorit, Mo = Montmorillonit, I = Illit, Mu = Muskovit

$c\{\}$ in Spuren vorhanden bzw. vermutet

a $\mathrm{Fe}_{\mathrm{HNO}}$ : $\mathrm{HNO}_{3}$-Auszug

* Zusammensetzung der beobachteten «Knollen"

** roter resp. schwarzer Bereich der beobachteten "Knollen»

Die mineralogische Zusammensetzung des Oberbodens wird weitestgehend durch Quarz, Feldspäte, Chlorit, Muskovit und Montmorillonit bestimmt. In größerer Bodentiefe präsentiert sich jedoch eine andere Ausgangslage. Knollenähnliche Fragmente im II-BCgg-Horizont sind ein typisches und häufiges Erscheinungsbild. Auf der einen Seite weisen diese «Knollen» eine dunkelbraune bis schwarze und auf der andern eine orange bis rote Farbe auf. Röntgendiffraktometrische Untersuchungen ergaben, daß es sich beim dunklen Material zur Hauptsache um Kaolinit und Quarz und beim rötlichen Substrat um Lepidokrokit $(\gamma-\mathrm{FeOOH})$ und Quarz handelt. Da die tieferen Bodenschichten als stark hydromorph bezeichnet werden können, scheint es als wahrscheinlich, daß das Vorkommen der Minerale Kaolinit und Lepidokrokit direkt oder indirekt an Redoxprozesse und damit gekoppelte Hydrolysereaktionen geknüpft ist. Nebst der Auflösung von Feldspäten und deren Umwandlung in Kaolinit (Gleichung 1) müssen demzufolge zwei weitere Prozesse berücksichtigt werden:

a) Auflösung von $\mathrm{FeOOH}$ :

$\mathrm{FeOOH}_{(\mathrm{s})}+\mathrm{e}^{-}+3 \mathrm{H}^{+}=\mathrm{Fe}^{2+}+2 \mathrm{H}_{2} \mathrm{O}$

b) Auflösung von Kaolinit:

$\mathrm{Al}_{2} \mathrm{Si}_{2} \mathrm{O}_{5}(\mathrm{OH})_{4(\mathrm{~s})}+6 \mathrm{H}^{+}=$

$2 \mathrm{H}_{4} \mathrm{SiO}_{4}(\mathrm{aq})+2 \mathrm{Al}^{3+}+\mathrm{H}_{2} \mathrm{O}$

FeOOH bildet sich unter eher oxidierenden Bedingungen, während die Auflösung unter Freisetzung von $\mathrm{Fe}^{2+}$ (Fig. 2) unter reduzierenden Verhältnissen erfolgt (Glei- chung 3). Der Prozess der Oxidation ist im allgemeinen mit saureren Verhältnissen in der Bodenlösung verbunden. Der pH-Wert des Bodens im Lepidokrokitdominierten Bereich ist tatsächlich tiefer $(\mathrm{pH}=5,4$, vgl. Tab. 4). Der Boden-pH in der Kaolinitzone, welche morphologisch dem reduktiven Bereich zugeordnet werden kann, beläuft sich auf 6,1. Ein höherer $\mathrm{H}^{+}$-Gehalt bedingt, daß Kaolinit verstärkt hydrolysiert wird (Gleichung 4). Reduzierende Verhältnisse in Pseudogleyen finden sich nach längeren oder stärkeren Niederschlagsereignissen zuerst in den Makroporen (präferentielle Fließwege). Eine beginnende Austrocknung des Bodens in niederschlagsfreien Perioden bewirkt wahrscheinlich, daß die Bodenlösung bezüglich der Festphase Kaolinit übersättigt wird und somit eine Ausfällung stattfinden kann. Die weitere Passage des Bodenwassers von den Makroporen in die Mikroporen hat vermutlich zur Folge, daß das Löslichkeitsprodukt von Kaolinit, aufgrund des tieferen $\mathrm{pH}$-Wertes, unterschritten wird und eine Ausfällung somit nicht mehr möglich ist. Im oxidativen Bereich sollte sich theoretisch Goethit $(\alpha-\mathrm{FeOOH})$ anstelle von Lepidokrokit $(\gamma-\mathrm{FeOOH})$ bilden. Wie Untersuchungen von KRISHNAMURTI \& HUANG (1993) belegen, fördert die Anwesenheit bereits geringer Mengen an organischen Liganden (beispielsweise Citrat) die Bildung von Lepidokrokit. Fehlen hingegen organische Liganden, wäre Goethit die stabile Phase bei pH-Bedingungen von 4,0-8,0. In der Kaolinitzone (schwarzer Bereich der Knollen) ist der pH-Wert, verursacht durch häufiger reduzierende Verhältnisse, erhöht. FeOOH ist in diesem Bereich jedoch nicht stabil. Lepidokrokit wird bei einem tiefen $\mathrm{p} \varepsilon$ unter Freisetzung von $\mathrm{Fe}^{2+}$ relativ rasch aufgelöst (vgl. Fig. 2). 


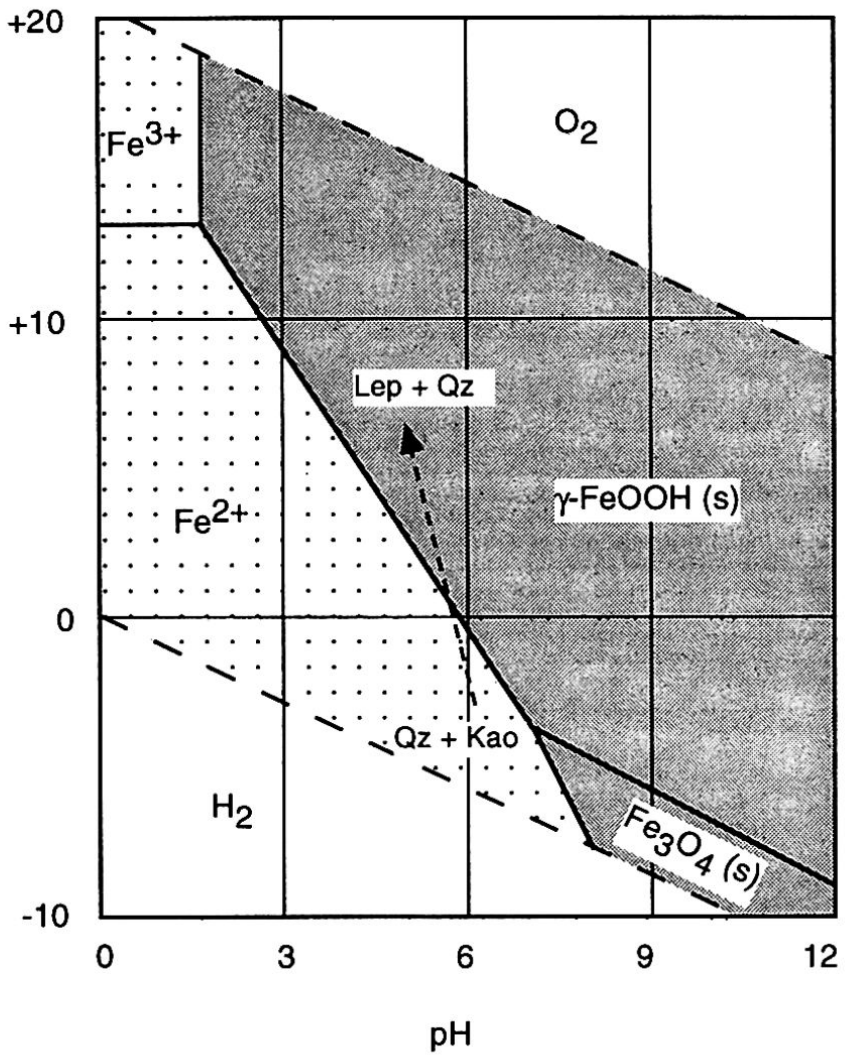

Fig. 2. $\mathrm{pH}-\mathrm{p} \varepsilon$-Stabilitätsdiagramm (bezogen auf $9^{\circ} \mathrm{C}$, thermodynamische Daten nach SIGG \& STUMM 1994) für die Lokalität Buchen. Die berücksichtigten Festphasen sind $\gamma$ $\mathrm{FeOOH}$ und $\mathrm{Fe}_{3} \mathrm{O}_{4}$ (Lep = Lepidokrokit, $\mathrm{KaO}=$ Kaolinit, $\mathrm{Qz}=$ Quarz). Getroffene Annahme: $\mathrm{Fe}^{2+}=10^{-5} \mathrm{M}$, total gelöster Kohlenstoff $\mathrm{C}_{\text {tot }}=10^{-3} \mathrm{M}$.

\section{Isola}

Aufgrund der Analysedaten (vgl. Profilverlauf von $\mathrm{Fe}_{\mathrm{d}}$, Tab. 5) könnte der Boden als Podsol charakterisiert werden. Die morphologische Ausprägung des Profils deutet jedoch eher auf einen Ranker hin, der in einer Tiefe von ca. $50 \mathrm{~cm}$ einem mit $\mathrm{Fe}$ angereicherten Horizont aufliegt. Der Korngrößenwechsel in dieser Tiefe ist markant, so daß die Vermutung einer nachträglichen Akkumulation des Hangenden nahesteht. Der wenig entwickelte Boden ist mäßig sauer mit $\mathrm{pH}-$ Werten im Bereich von 4,5-5,6 (Tab. 5). Röntgendiffraktometrische Untersuchungen zeigen, daß Kaolinit in $105 \mathrm{~cm}$ Tiefe z. T. in größeren Mengen vorhanden ist. Im IsolaDelta stellt sich die Frage, ob hydrothermale Prozesse die Bildung von Kaolinit (als Bestandteil des Primärgesteins) eventuell bewirken konnten. Petrographische und geologische Untersuchungen geben jedoch keinen Hinweis auf Kaolinit im Primärgestein (LINIGER \& GUNTLI 1988; SPILlMANN 1993). Die Gesteine der Umgebung weisen zudem einen Metamorphosegrad auf, welcher der Grünschiefer- und Amphibolitfazies zuzuordnen ist. Gemäß CAIRONI \& TROMMSDORFF (1988) ist Kaolinit unter solchen Bedingungen nicht stabil. Kaolinit wäre bereits bei niedrigeren Temperaturen in Pyrophyllit umgewandelt worden.

Der untersuchte Boden ist des weitern durch den Einfluß des Grundwassers gekennzeichnet. Der Grundwasserspiegel schwankt hauptsächlich in einem Bereich von 50-70 cm Tiefe (GEHRIG 1996). Unterhalb des Grundwasserspiegels lassen sich signifikante Mengen an Kaolinit vorfinden. Somit ergibt sich die Vermutung, daß das Grundwasser einen direkten Einfluß auf die Bildung von Kaolinit ausübt. Aufgrund des lokalen Bodenchemismus scheinen die entsprechenden Bedin-

Tabelle 5 Bodeneigenschaften der Lokalität Isola

\begin{tabular}{llllccccr}
\hline $\begin{array}{l}\text { Tiefe } \\
(\mathrm{cm})\end{array}$ & $\begin{array}{l}\text { Horizont- } \\
\text { bezeichnung }\end{array}$ & $\begin{array}{l}\mathrm{pH} \\
\left(\mathrm{CaCl}_{2}\right)\end{array}$ & $\begin{array}{l}\mathrm{Fe}_{\mathrm{d}}{ }^{\mathrm{d}} \\
\mathrm{g} \mathrm{kg}^{-1}\end{array}$ & $\mathrm{~T}(\%)$ & $\begin{array}{c}\text { Körnung } \\
\mathrm{U}(\%)\end{array}$ & $\mathrm{S}(\%)$ & Mineralogie $^{\mathrm{b}}$ & $\begin{array}{c}\text { Alter (yBP) } \\
\mathrm{PF}^{\mathrm{c}}\end{array}$ \\
\hline 10 & Ah & 4,5 & 3,3 & 3 & 25 & 72 & & \\
30 & (B)C & 4,8 & 2,4 & 3 & 17 & 80 & Qz, Kfsp, Plag, Mu, Chl & 1050 \\
46 & (B)C & 5,0 & 3,2 & 3 & 40 & 57 & & 1050 \\
50 & II Bfe & 4,9 & 7,9 & 10 & 70 & 20 & & 1145 \\
65 & II Bfe & 5,1 & 6,2 & 25 & 60 & 15 & & 1350 \\
105 & II Cg & 5,0 & 4,7 & 15 & 65 & 20 & Qz, Kao, Mo, Mu, Plag, Kfsp & 1500 \\
130 & III C & 5,6 & 2,3 & 2 & 8 & 90 & & 1575 \\
233 & Aa & 4,6 & n.b. & n. b. & n.b. & n.b. & & 1650 \\
\hline
\end{tabular}

a $T=$ Tonfraktion $(<2 \mu \mathrm{m}), U=$ Siltfraktion $(2-63 \mu \mathrm{m})$ und $S=$ Sandfraktion $(63-2000 \mu \mathrm{m})$

b $\mathrm{Qz}=$ Quarz, Plag = Plagioklas, Kfsp = Kali-Feldspat, Mo = Montmorillonit, Mu = Muskovit, Kao = Kaolinit, Chl = Chlorit

c Alter aufgrund der Pollenfrequenz

a $F e_{d}$ : Dithionit-Auszug 
gungen für eine Kaolinitgenese zu bestehen. Die gemessenen $\mathrm{Al}-$, Si- und $\mathrm{H}^{+}$-Konzentrationen im Wasserauszug (nicht tabelliert) des Bodenmaterials in $105 \mathrm{~cm}$ Tiefe belegen, daß die wäßrige Phase bezüglich der Kaolinitfestphase übersättigt ist $(\Omega=+0,8$ bis $+3,2)$. Es erscheint somit als wahrscheinlich, daß Kaolinit an Ort und Stelle ausgefällt wird.

Organisches Material in ca. $65 \mathrm{~cm}$ und ca. $230 \mathrm{~cm}$ Tiefe konnte radiometrisch datiert werden (GEHRIG 1996). Die erhaltenen ${ }^{14} \mathrm{C}$-Alter von $1145 \pm 55$ yBP für $65 \mathrm{~cm}$ Tiefe und $1575 \pm 55$ yBP für $230 \mathrm{~cm}$ sowie das errechnete Alter über die Pollenfrequenz erlauben die Hypothese, daß Kaolinit auch in vergleichsweise sehr jungen Sedimentablagerungen bzw. Böden vorkommen kann und rezenten Bildungsprozessen zuzuschreiben ist.

\section{Die Untersuchungsresultate im überregionalen Rahmen}

Um den momentan rein lokalen Bezug der Ergebnisse in einem etwas weiteren Rahmen zu sehen, wurden Resultate aus bodenkundlichen und tonmineralogischen Untersuchungen (sofern vorhanden) der jeweils weiteren Umgebung in einen Gesamtvergleich mit einbezogen.

JÄGGLI (1968) untersuchte diverse Bodenprofile (nur Tonfraktion, Parabraunerden) auf verschiedenen Schotterablagerungen (Muttenz, Möhlin, Rütihard und Neuwiler) im Rheintal unterhalb von Möhlin. Bei diesen Schotterablagerungen handelt es sich um ältere und jüngere Deckenschotter (ca. 600000 yBP resp. ca. $470000 \mathrm{yBP}$ ) und um Hoch- und Niederterrassenschotter (ca. 240000 yBP resp. ca. 120000 yBP). Die weitaus dominanten Minerale der Tonfraktion sind Bodenchlorite, Illite sowie glimmerartige und vermikulitartige Mineralien. Kaolinit ist von untergeordneter Bedeutung. Bedeutende Unterschiede zwischen den einzelnen Bodenprofilen bezüglich ihres Kaolinitgehalts wurden nicht ausgemacht. Ein geringer Gehalt an Kaolinit in Parabraunerden auf relativ gut durchlässigem Ausgangsmaterial (Lokalität Seeliboden und Chräbis) wird somit auch an Vergleichsstandorten bestätigt. Eine aktive Kaolinitbildung scheint an diesen Standorten ebenfalls nicht der Fall zu sein.

Aktive neuzeitliche Bildungen von Kaolinit sind in normal perkolierten Böden auf Kalkgestein (Jura) meist kaum nachweisbar. STICHER (1969) und VÖKT (1974) zeigen sogar, daß Kaolinit bei der Freisetzung aus dem Kalkstein abgebaut werden kann. Höhere Kaolinitgehalte (um ca. 30\%, bezogen auf die Feinerde), die möglicherweise eine rezente Bildung nicht ausschließen, finden sich in vernäßten Böden (BGS 1979, РOCHON 1979) auf mergeligem Malmkalk (Argovien), z. B. in der Nähe der Combe des Begnines (Kanton Waadt). Böden auf Opalinuston, welcher lithostratigraphisch im Juragebirge die unterste Doggereinheit bildet, weisen z. T. recht hohe Kaolinitgehalte auf (20-30\%). Dies scheint durch den bereits hohen Kaolinitgehalt des Ausgangsmaterials bedingt zu sein. SCHWER (1994) zeigt anhand von Verwitterungsbilanzen, daß in den Übergangshorizonten (Sd-Cv) pseudogleyiger BraunerdePelosolen offenbar ideale Voraussetzungen für eine rezente Bildung von Kaolinit herrschen.

Das Vorkommen von Kaolinit in Böden (auf Silikatgestein) alpiner Regionen wurde auch von DREHER (1994) beschrieben. Bei diesen Böden handelt es sich vorwiegend um alpine Pseudogleye auf Biotitschiefer (Hohe Mut, im Ötztal). Das Alter der in dieser Arbeit untersuchten Pseudogleye beläuft sich auf max. 10000 Jahre. Dreher vermutet, daß die Kaolinitanteile dieser Böden als rezente Bildungen betrachtet werden können. Die Gegenwart von Kaolinit in den Hochgebirgsböden des Ötztals unterstützt somit die Hypothese, daß die Bildung von Kaolinit nicht zwangsläufig an das Vorherrschen warmer Klimabedingungen gebunden sein muß. Untersuchungen von ZABOWSKI \& UGOLINI (1992) an Hochgebirgsböden bestätigen, daß auch in einem Podsol unter sehr feuchten $(270 \mathrm{~cm}$ Jahresniederschlag) und kalten Bedingungen das Löslichkeitsprodukt von Kaolinit häufig überschritten werden kann.

\section{Schlußfolgerungen}

Bei der Interpretation obiger Resultate muß an die relativ geringe Probenzahl erinnert werden: durch den Miteinbezug von Literaturdaten läßt sich dennoch ein erster Quervergleich bewerkstelligen. Daraus leiten wir nachfolgende Hypothesen über die Bildungsbedingungen und somit das Vorkommen von Kaolinit in Böden der gemäßigten bis subalpinen/alpinen Klimate der Schweiz ab.

Kaolinit kann in relikten und sehr alten Böden (3758 Mio. yBP), die im wesentlichen unter subtropischen bis tropisch feuchten Klimaten entstanden sind, in recht großen Mengen nachgewiesen werden. Das Auftreten von Kaolinit in einer Terra fusca entspricht somit vollumfänglich den theoretischen Erwartungen. In normal perkolierten, rezenten Böden, die sich seit dem Zurückschmelzen der Gletscher nach der Maximalausdehnung der letzten Eiszeit (ca. 20000 yBP) entwickelten, kann unter gemäßigt-humiden Bedingungen im allgemeinen nur sehr wenig Kaolinit gefunden werden. Eine aktive Kaolinitbildung ist eher auszuschließen.

Der Wasserhaushalt eines Bodens scheint einen entscheidenden Einfluß auf die Bildungsbedingungen von Kaolinit zu haben. Häufig wird davon ausgegangen, daß eine intensive Perkolation die Bildung von Kaolinit begünstigt. Unsere Ergebnisse lassen eher den Schluß zu, daß eine gehemmte Perkolation (wie Stauwassereinfluß) oder Grundwasser die Kristallisation von Kaolinit - auch in nicht besonders niederschlagsreichen Regionen - ermöglicht. Die Kontaktzeit mit der Matrix und die Elektrolytkonzentration im Wasser scheinen vor allem in stauwassergeprägten oder grundwasserbeeinflußten Böden derart zu sein, daß bessere Bedingungen 
für ein chemisches Gleichgewicht nach Gleichung (1) bestehen. Dies erklärt, wieso Kaolinit auch in vergleichsweise jungen Böden und zusätzlich unter subalpinem Klima vorzufinden ist.

Grundwasser- oder Stauwassereinfluß im Boden geht mit Redoxprozessen einher. Oxidierende Bedingungen sind mit einer Freisetzung von Säuren und reduzierende mit einer entsprechenden Neutralisation verbunden. Reduzierende Bedingungen in sauren Böden führen da$\mathrm{zu}, \mathrm{da} ß$ der $\mathrm{pH}-$ Wert des Bodens und der Bodenlösung nicht allzu tief absinkt. Eine längere Aufenthaltszeit des Perkolats in der entsprechenden Bodenschicht bzw. ein intensiver Kontakt zwischen der Bodenmatrix und der Bodenlösung begünstigen möglicherweise die Kaolinitgenese. Oxidierende Bedingungen im Boden können das chemische Milieu (z. B. pH-Wert), zumindest auf ganz lokaler Ebene, derart verändern, daß das Löslichkeitsprodukt von Kaolinit unterschritten wird - mit der Konsequenz, daß eine Ausfällung verunmöglicht wird.

\section{Literatur}

BARSHAD, I. (1966): The effect of a variation in precipitation on the nature of clay mineral forming in soils from acid and basic igneous rocks. Internat. Clay Conf. (Jerusalem), Proc. vol. 1, 167-173.

BIRKELAND, P. W. (1984): Soils and geomorphology. Oxford University Press, Oxford.

BODENKUNDLICHE GESELLSCHAFT DER SCHWEIZ (BGS) (1979): Excursion pédologique dans le Haut-Jura. Exkursionsbeilagen, unpubl.

CAIRONI, V. \& TROMMSDORFF, V. (1988): Lezioni di petrologia delle rocce metamorfiche. Cooperativa libraria universitaria del politecnico, Milano.

DREHER, P. (1994): Tonmineralbildung in Braunerden aus Amphibolit und Biotitschiefer sowie Untersuchungen zur Biotitumwandlung im Modellversuch. Dissertation, Technische Universität München-Weihenstephan.

EGLI, M. (1995): The influence of increased $\mathrm{NH}_{4}+$ deposition rates on aluminium chemistry and alkalinity in the percolate of acid soils. Dissertation, Universität Zürich.

ESTEOULE-CHOUX, J. \& BLANCHET, C. (1987): L'altération directe de Muscovites et de Biotites en Halloysite: Mise en évidence par microscopie électronique à balayage. Clay Minerals 22, 11-20.

FITZE, P., CAPAUL, U., MEULI, R. \& ACHERMANN, M. (1989): Landschaftsökologisches Forschungsprojekt "Gubrist": Ergebnisse der Beobachtungsperiode 1983-1988. Schriftenreihe Physische Geographie 29, Zürich.
GEHRIG, S. (1996): Stratigraphische, pedologische und geomorphologische Untersuchungen am Isola-Delta. Abfolge, Intensität und Häufigkeit von Ereignissen. Unpubl. Diplomarbeit, Geographisches Institut, Universität Zürich.

HEIM, D. (1990): Tone und Tonminerale: Grundlagen der Sedimentologie und Mineralogie. Enke Verlag, Stuttgart.

JÄGGLI, F. (1968): Mineralogische und chemische Untersuchungen der Tonfraktion aus Böden von Rheinschottern verschiedenen Alters. Dissertation ETH Zürich, Nr. 4149.

KRISHNAMURTI, G.S.R. \& HUANG, P.M. (1993): Formation of lepidocrocite from iron (II) solutions: stabilization by citrate. Soil Sci. Soc. Am. J. 57, 861-867.

KÜHNEN, H. (1984): Morphogenese des Möhliner Feldes (Hochrheintal). Materialien zur Physiogeographie 5, 43-51.

LINIGER, M. \& GUNTLI, P. (1988): Bau und Geschichte des zentralen Teils der Margna-Decke. Schweiz. Mineral. Petrogr. Mitt. 68, 41-54.

MURRAY, H. H. \& KELLER, W.D. (1993): Kaolins, kaolins, and kaolins. In: Kaolin genesis and utilization. (Ed. by MURRAY, H.H., BUNDY, W.M., HARVEY, C. C.). The Clay Minerals Society, Special publication No. 1, 1-24.

PLUMMER, L.N., JONES, B. F. \& TRUESDELL, A. H. (1976): WATEQF - A FORTRAN IV version of WATEQ, a computer program for calculating chemical equilibrium of natural waters. U.S. Geological Survey Water-Resources Investigations 76-13.

POCHON, M. (1979): Origine et évolution des sols du HautJura suisse: phénomènes d'altération des roches calcaires sous climat tempéré humide. Denkschriften der Schweizerischen Naturforschenden Gesellschaft XC, Zürich.

SCHWER, P. (1994): Untersuchungen zur Modellierung der Bodenneubildungsrate auf Opalinuston des Basler Tafeljura. Physiogeographica 18. Dissertation, Universität Basel.

SIGG, L. \& STUMM, W. (1994): Aquatische Chemie: Eine Einführung in die Chemie wäßriger Lösungen und natürlicher Gewässer. Verlag der Fachvereine Zürich und Teubner Verlag, Zürich und Stuttgart.

SPILLMANN, P. (1993): Die Geologie des penninisch-ostalpinen Grenzbereichs im südlichen Berninagebirge. Dissertation ETH Zürich, Nr. 10175.

STICHER, H. (1969): Reaktion von Kaolinit mit Kaliumhydroxid. Habilitationsschrift, ETH Zürich.

STUMM, W. (1992): Chemistry of the solid-water interface: processes at the mineral-water and particle-water interface in natural systems. Wiley-Interscience Publication, New York. VELDE, B. (1992): Introduction to clay minerals: chemistry, origins, uses and environmental significance. Chapmann \& Hall, London.

VÖKT, U. (1974): Umwandlung der Silikate bei der Bodenbildung aus Kalkstein auf dem Geißberg bei Villigen AG. Dissertation ETH Zürich, Nr. 5003.

WINISTÖRFER, D. (1993): Analytisch-methodische Untersuchungen zur Speziierung verschiedener Schwermetalle in Extraktions-Bodenlösungen. Dissertation, Universität Basel. ZABOWSKI, D. \& UGOLINI, F.C. (1992): Seasonality in the mineral stability of a subalpine spodosol. Soil Science 154 , 497-507. 